\title{
Was zeichnet gute Bildungsarbeit aus? - Die neuen VENRO-Qualitätskriterien für entwicklungspolitische Bildungsarbeit bieten Orientierungshilfe
}

Im Frühjahr 2020 begann die VENRO Arbeitsgruppe Bildung Lokal/Global die „Qualitätskriterien für entwicklungspolitische Bildungsarbeit“ zu überarbeiten. Die Qualitätskriterien waren 2012 als Gemeinschaftswerk zahlreicher Bildungsakteur/-innen aus Zivilgesellschaft und Bildungseinrichtungen entstanden. Da sich die Diskurse innerhalb der Bildungsszene in den vergangenen zehn Jahren maßgeblich weiterentwickelt haben, wurden die Qualitätskriterien nun tiefgreifend überarbeitet. Insbesondere machtkritische und postkoloniale Perspektiven und die Erkenntnisse der Studie „Wirkungsorientierung in der entwicklungspolitischen Inlandsarbeit" fanden Eingang.

Die Qualitätskriterien sind inklusiv formuliert und können dadurch nicht nur von Akteur/-innen genutzt werden, die sich der entwicklungspolitischen Bildungsarbeit zuordnen. Sie können auch Bildungspraktiker/-innen aus dem Globalen Lernen, der Bildung für Nachhaltige Entwicklung, der Global Citizenship Education und anderen artverwandten, wertebasierten Bildungskonzepten Orientierungshilfe leisten. In vier Kapiteln, bietet die Publikation den Bildungspraktiker/-innen Unterstützung bei (1) der Planung und Organisation, (2) der inhaltlichen Gestaltung und (3) der didaktischen und methodischen Umsetzung von Bildungsangeboten. Kapitel 4 setzt sich zudem speziell mit Bildungsangeboten in Kooperation mit schulischen Partner/-innen auseinander und dient als Beispiel für die Anwendung der allgemeinen Kriterien auf einen konkreten Bildungsbereich.

Zentraler Angelpunkt der Qualitätskriterien ist ein kritisches Entwicklungsverständnis. Globale Zusammenhänge und ihre Auswirkungen auf lokale Situationen, globale Abhängigkeiten und Machthierarchien sollen in den Blick genommen werden. Dabei helfen postkoloniale, rassismuskritische und intersektionale Ansätze. Sie sensibilisieren beispielsweise dafür, beim Versuch, komplexe, globale Sachverhalte zugänglich und verständlich zu machen, auf vereinfachende Darstellungen und die Unterteilung zwischen einem „wir“ und den ,anderen“ zu verzichten, sowie die eigene Quellenwahl kritisch zu hinterfragen. Auch ein Blick in die Geschichte auf historische Ursprünge globaler Ungleichheiten ist zentral. Er hilft den Lernenden, aktuelle Zustände nicht als natürlich gewachsen, sondern als Ergebnis politischer Entscheidungen und Handlungen zu verstehen und eröffnet damit Möglichkeiten zur Veränderung.

Sowohl bei der Vorbereitung und Organisation als auch bei der Auswahl der didaktischen Methoden steht die Zielgruppenorientierung im Mittelpunkt. Durch eine intensive Auseinandersetzung mit der Zielgruppe, ihren Bedarfen, Vorkenntnissen und ihrer Lebenswelt können Faktoren mit einbezogen werden, welche die Wirksamkeit des Bildungsangebots direkt beeinflussen. Das betrifft beispielsweise die Auswahl des Veranstaltungsortes, der Lehrmethoden oder auch die Themensetzung. Gleichzeitig muss eine machtkritische Betrachtung auch auf die Bildungsanbietenden selbst bezogen werden: Versteht sich die Lehrkraft selbst auch als Lernende? Wer war an der Konzeption des Bildungsangebots beteiligt und wer war davon ausgeschlossen?

Kritische Diskurse brauchen Raum, um sich weiterzuentwickeln. Die Qualitätskriterien sollen als Beitrag zu einer lebendigen und offenen Debatte über Qualität und Wirksamkeit in der entwicklungspolitischen Bildung verstanden werden, die auch in Zukunft weitergeführt werden muss. Sie sind kein Pflichtenheft, das von Bildungspraktiker/-innen eins zu eins abgearbeitet werden muss. Wie Bildungsangeboten wirkungsvoll gestaltet werden können, hängt stark vom jeweiligen Kontext ab. Die Qualitätskriterien sollen hier als Orientierungshilfe dienen und in erster Linie einen Reflexionsprozess anregen. Sie helfen dabei, das Augenmerk auf bestimmte wichtige Faktoren für die Wirksamkeit von Bildungsangeboten zu legen, über die bei Planung und Vorbereitung, Durchführung und Nachbereitung von Bildungsangeboten aktiv nachgedacht und die im Team besprochen werden sollen. Zur praktischen Anwendung der Kriterien dienen die angeschlossenen Leitfragen. Sie geben beispielhaft Anregung dazu, welche Fragen sich Bildungspraktiker/-innen zur Überprüfung der Qualitätskriterien stellen können.

Die „VENRO-Qualitätskriterien für entwicklungspolitische Bildungsarbeit" stehen auf der VENRO-Webseite zum kostenlosen Download zu Verfügung: https://venro.org/filead min/user_upload/Dateien/Daten/Publikationen/Handbuch/ VENRO_Qualit\%C3\%A4tskriterien_Bildungsarbeit_2021.pdf. Bei Interesse an einem Print-Exemplar wenden Sie sich gerne an veranstaltungen@venro.org.

Lara Fedorchenko,

VENRO - Verband Entwicklungspolitik und Humanitäre Hilfe doi.org/10.31244/zep.2021.03.07 